\title{
Prenatal screening for congenital toxoplasmosis in Campania: preliminary report on activities and results
}

\author{
L Stagni', MA Romano', A Romano', A Magli², F Briganti ${ }^{3}$, MA Del Pezzo ${ }^{4}$, W Buffolano ${ }^{1 /+}$ \\ ${ }^{1}$ Departments of Paediatrics ${ }^{2}$ Pediatric Ophthalmology ${ }^{3}$ Radiodiagnostics ${ }^{4}$ Microbiology Labs, University Hospital Federico II, \\ via Pansini 5, 80131 Naples, Italy
}

By 1997, an open cohort of 1,652 live newborn of 1,637 mothers with gestational toxoplasmosis had been recruited in the Campania region to monitor the burden of congenital toxoplasmosis (CT). Of the 1,556 mother-child pairs that completed the follow up, 92 definite cases were detected, yielding a 5.9\% (4.8-7.1 95\% CI) transmission rate. The onset was patent for $43 \%$ of patients and sensorineural complications were shown for a further $15 \%$ of subclinical onset patients later than two years of age. The overall prevalence of toxoplasmosis during gestation was 2.46 of 1,000 deliveries, while the prevalence of definite CT was 1.38 of 10,000 live newborns. However, there is still room for intervention, as only $23 \%$ of the maternal diagnoses were proven through seroconversion, 63 of the lategestation seroconverters remained untreated, and six probable CT diagnoses were made following referrals due to patent sequelae and born during the study period. There was a positive secular trend on the rates of infant referral and definite CT diagnosis, according to the live birth rate $\left(\chi^{2}\right.$ for trend $\left.<0.001\right)$. Extension of this surveillance system across the country could help to define a future strategy for prevention.

Key words: gestational prevalence of toxoplasmosis - prevalence of congenital toxoplasmosis - prenatal screening

Although toxoplasmosis is considered a benign disease for immunocompetent adults, toxoplasmosis transmitted to the foetus during gestation can have dramatic consequences.

By the 1990s, pressure to develop a strategy for fighting congenital toxoplasmosis (CT) reached its peak, and Toxo-testing during gestation became widespread in $\mathrm{Eu}-$ rope for both screening and non-screening countries (Leroy et al. 2005). Beginning in 1994, the Italian Society of Perinatal Medicine recommended tri-monthly testing of seronegative pregnant women and treatment of IgM positive cases with spiramycin (Bagnoli et al. 1994). By 1995, the National Health System warranted charge-free access and tri-monthly testing of seronegative pregnant women (GURI 1995) and, in 1998, the testing schedule was changed to monthly.

Unfortunately, baseline information sets and surveillance systems are still missing at the national level. The Regional Health Bureau launched a monitoring system (Perinatal Infection Register) for the CT disease burden and clinical practices in Campania in 1997. This pilot area was chosen because of its high birth rate $(11 \%$ of the national rate), medium-high seroprevalence among delivering women (40.2\%) (Buffolano et al. 1996) and widespread prenatal Toxo-testing (Buffolano et al. 1994). This study outlines the preliminary data collected during the first ten years of the pilot programme.

Financial support: Regional Health Bureau (DGR 10788 del 31.12.1996)

+Corresponding author:wilma@unina.it

Received 10 October 2008

Accepted 24 March 2009

\section{PATIENTS, MATERIALS AND METHODS}

The Perinatal Infection Register is an open observational cohort study involving a network of 67 delivery hospitals (RePuNaRC, covering $89 \%$ of local births) and the Paediatric Regional Referring Centre (PRRC) located at University Hospital Federico II of Naples, which is the main city of Campania.

Briefly, a trained neonatologist in each delivery hospital referred any live newborn of mothers with proven (IgG seroconversion based on at least 2 post-conception serum samples taken at least 3 weeks apart) or unproven [positive IgM and IgG and increasing IgG level and/or positive IgA and/or exclusion of high IgG avidity test before a gestational age (GA) of 12 weeks] gestational toxoplasmosis to the PRRC for postnatal management. Following a proven diagnosis, the GA at infection was considered undetermined if the interval between the last negative and the first positive sample exceeded 12 weeks. When necessary, the circumstances and GA at maternal diagnosis and the prenatal management were further investigated through a structured interview, reevaluation of the gestation charts and a telephone interview of the personal obstetrician upon PRRC enrolment. Based on the precision of the maternal diagnosis (proven or unproven) and the GA at infection (presumed or undetermined), different management programmes were offered to newborn infants eligible for CT diagnosis. Infants of mothers with unproven diagnoses entered the (B) programme, consisting of testing at zero, six and 12 months of age, indirect fundoscopic examination and cerebral ultrasonography (once). Further testing was carried out only if clinically indicated or if infants remained positive beyond six months of age for specific IgG. Infants of mothers with proven primary toxoplas- 
mosis during gestation entered the (A) programme, which included monthly serological follow up (EIA IgM and $\operatorname{IgG}$, ISAGA IgM and EIA IgA) until 12 months of age, cytomegalovirus (CMV) culture and/or Polymerase Chain Reaction (CMV- PCR) to exclude congenital CMV (once), cerebral ultrasonography and computed tomography (CT scan) and indirect fundoscopic examination (monthly until 6 months of age). For late enrolment, positive CMV-PCR results were confirmed by repeating the test on blood spots collected for mandatory phenylketonuria screening. Auditory Brain Reaction and EEG were carried out at least once. Newborns whose mothers seroconverted after 31 weeks GA were treated with tertiary prevention, including three weeks of pyrimethamine, sulphadiazine and folinic acid treatment. Twelve months of a high dose, continuous treatment was applied in the following conditions: (i) positive IgM and/or IgA (2 samples taken 2 weeks apart); (ii) lack of monthly decay or an increase in specific IgG level over time ( 2 samples taken 2 weeks apart); (iii) acute retinochoroiditis; (iv) cerebral microcalcification with or without hydrocephalus (after exclusion of congenital CMV). A CT diagnosis was considered definite or excluded beyond 12 months of age according to the definite persistence or disappearance of specific IgG. The infants with definite CT were re-examined (clinically, fundoscopically and neurologically) at least twice a year up to six years and once a year, thereafter.

\section{RESULTS}

Impact - Cohort recruitment and the burden of CT in the Campania region are shown in Fig 1. From 19972007 , there were 1,742 newborn infant referrals. Ninety infants $(5 \%)$ were considered ineligible as maternal toxoplasmosis during gestation was excluded (persistent, residual IgM for 28 patients and false IgM for 62 patients). In total, 1,652 infants (including 15 twin couples) of 1,637 mothers [referral range 113-178, mean (M) 148 infants per year] were enrolled and 92 definite CT (range $2-14$, M 8 per year) cases were detected. Eighty-one infants ( $11 \%$ from seroconverting mothers) were lost at the follow up, yielding a definite diagnosis rate of $95.1 \%$. There were eight proven cases of CT in twins, six in concordant twins and two in discordant twins. The onset was patent for 40 of the cases $(43 \%)$, benign (i.e., nonmacular eye involvement and/or cerebral microcalcification, without hydrocephalus) for 24 (26\%), severe (i.e., macular retinochoroiditis and/or hydrocephalus) for 16

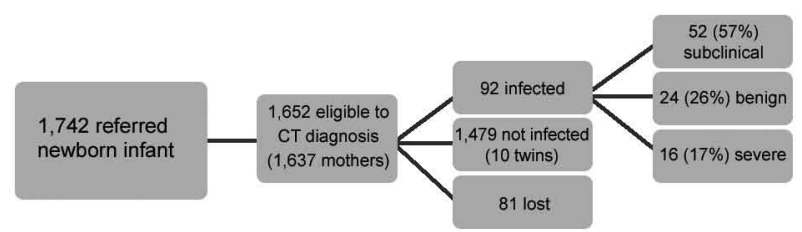

Fig. 1: burnden of congenital taxoplasmosis on Campania region of Italy (report from "Perinatal Infection on Register", period 1997-2007).
(17\%) and subclinical for $52(57 \%)$. The study identified 18 infants with eye involvement (20\%) and $39(42 \%)$ with cerebral abnormalities (isolated or hydrocephalus associated calcifications). The 92 infected patients received 12 months of high dose, continuous treatment; 11 (12\%) patients were given twice the folinic acid dosage, and two $(2 \%)$ patients received half doses of pyrimethamine because of bone marrow suppression. One case had a temporary suspension ( 3 weeks) of treatment due to an episode of simple partial seizures associated with fever. Four infants with symptomatic onsets had chronic seizures requiring treatment, three had a severe onset with ventricular dilatation and one had a benign onset. Three of the severe onset patients demonstrated neurodevelopmental delays. Two of the benign onset cases were lost before 24 months of age; the first was an irregular Chinese immigrant and the second was a native infant who died of measles complications. Based on the local birth rate (range 62,599-71,972, M 66,431), the overall prevalences of toxoplasmosis during gestation (including both proven and unproven diagnoses) and of definite CT were 2.46 in 1,000 deliveries $(2.34-2.58,95 \% \mathrm{CI})$ and 1.38 in 10,000 live newborns $(1.10-1.67,95 \% \mathrm{CI})$, respectively. There was an increasing prevalence of sequelae (mainly ocular) with time (follow up range 24 months to 10 years, M 5.7 year). At 24 months of age, 11 of 74 (15\%) patients without retinochoroiditis at onset demonstrated ocular involvement (6/52 subclinical onset; 4/17 isolated microcalcification onset; $1 / 3$ microcalcification and hydrocephalus onset); there were no significant differences according to the type of onset. One patient with a severe onset and congenital CMV coinfection developed an auditory impairment. Another patient developed a severe bilateral visual impairment. The distribution of symptoms at onset and at two years of age is shown in Fig. 2.

Prenatal screening performance - Overall, 374 of $1,637(23 \%)$ maternal diagnoses were proven based on seroconversion. For 17 of the mothers $(4 \%$ of proven
Distribution of findings at onset

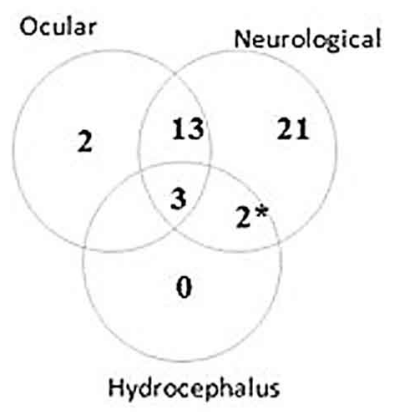

Distribution of findings during the follow up period (ranging 18 months to 10 years $)^{a}$

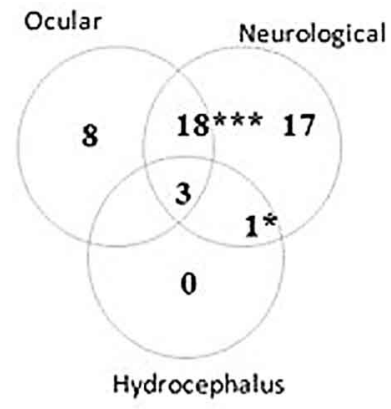

Fig. 2: distribution of main finding on 92 definite, born between January 1997-December 2007. $a$ : two benign onset patients were lost to follow up before age 18 months; Asterisk means the number of patient with seizures requiring chronic treatment in a category. 
seroconversion cases), the time of infection was undetermined and three delivered an infected child. Secondary prevention was instituted for 1,566 (96\%) of the IgM-IgG-positive mothers. This consisted of continuous spiramycin for 1,527 (93\%) and an alternating therapy of pyrimethamine-sulphadiazine combination and spiramycin for $39(1 \%)$ of 374 seroconverters. Among the 65 untreated mothers, there was one irregular Chinese immigrant, one native with gastrointestinal complications following spiramycin treatment and 63 who seroconverted late in gestation ( $\geq 30 \mathrm{GA})$. The transmission rates were $5.9 \%(4.8-7.195 \% \mathrm{CI}), 21 \%(17-2695 \% \mathrm{CI})$ and $0.8 \%(0.4-1.595 \% \mathrm{CI})$ for total diagnoses, proven seroconversion and unproven/indeterminate GA at infection, respectively. During the study period, six children with probable CT were identified following belated referrals (after 10 months of age) and excluded from the analysis. All six were identified following ocular complications, such as strabismus (3), nystagmus and microcornea (3); cerebral calcifications were visible in brain CT scans and maternal serology was compatible with toxoplasmosis during gestation. There was a positive secular trend in referrals and the definite $\mathrm{CT}$ diagnosis rate, according to the live birth rate $(\chi 2$ for trend $<0.001$ ).

\section{DISCUSSION}

A measure of the toxoplasmosis disease burden in humans and animals is badly needed (EFSA 2007). Health care demands for CT are high, despite the undemonstrated effectiveness of prenatal and newborn screening, poor treatment options and difficulties associated with randomised treatment controlled trials, including ethical barriers and sample size (Thiebaut et al. 2006). A surveillance system focused on newborns considered at risk has been implemented in the Campania region. The evaluation of Toxo-testing practices, reporting and referral of any suspected infant for diagnosis to the one PRRC for disease management and inclusion on the Perinatal Infection Register was considered feasible, cheap and well-accepted, as more than $95 \%$ of delivering women are inpatient for more than $48 \mathrm{~h}$. The burden of gestational toxoplasmosis complications, including abortion and termination, were considered to be of low relevance for rough measures of clinical and economic costs.

The burden measures gave conflicting results (Lebech et al. 1996). According to the local birth rate, the overall prevalence of defined CT was within the low incidence rate category for EU countries (1.38 per 10,000 live births versus 1-15 per 10,000 live births) (Gilbert \& Peckham 2002), but the overall prevalence of toxoplasmosis during gestation was found to be as high as 2.46 per 1,000 deliveries.

However, the overall prevalence of $\mathrm{CT}$ may be underestimated as there were several instances of belated CT diagnosis based on sequelae and the literature indicates that there is a high prevalence of symptomatic onset of disease (SYROCOT 2007). It is noteworthy that both the prevalence of toxoplasmosis-complicated pregnancies, which end in termination or abortion, and the preva- lence of neglected CT cases remain unknown. Further analyses could examine the effects of inequalities on the time schedule and frequency of testing for onset severity definitions. In fact, a testing schedule focused on the first $2 / 3$ of the gestational period is consistent with a low transmission rate and a high clinical severity rate (Fig. 1) (EMSCOT 2003). In addition, cerebral CT scans and indirect fundoscopy may be more accurate for detecting brain and retinal lesions compared to ultrasonography and direct fundoscopy.

However, concerns of litigation and a low practice in preconception (or very early during gestation) testing could result in the over-treatment of toxoplasma IgMIgG-positive pregnant patients for whom toxoplasmosis during gestation cannot be excluded. In fact, treatment was carried out for 1,261 unproven maternal diagnoses.

A positive secular trend in infant referrals due to maternal toxoplasmosis during gestation may be a reflection of improved access to testing, though prenatal screening rates remain low. In fact, less than $30 \%$ of the maternal diagnoses relied on proven seroconversion, $12 \%$ of the mother-to-child transmissions occurred when the maternal diagnosis was unproven/undetermined GA at infection, and some symptomatic CT cases were identified by patent sequelae.

Access to free screening and good Toxo-testing attitudes may jump-start screening, but its efficacy remains questionable where adequate infrastructure is still missing.

In light of concerns regarding the effectiveness and feasibility of prenatal screening, extension of this monitoring system across the country could help to define a future strategy for the prevention of CT.

\section{REFERENCES}

Bagnoli F, Buffolano W, Carosi G, Cataldi G, Martinelli P 1994. Raccomandazioni per la prevenzione, diagnosi e terapia prenatale e neonatale della toxoplasmosi. Boll Soc It Med Perinat 1: 4-19.

Buffolano W, Gilbert RE, Holland FJ, Fratta D, Palumbo F, Ades AE 1996. Risk factors for recent Toxoplasma infection in pregnant women in Naples. Epidemiol Infect 116: 347-351.

Buffolano W, Sagliocca L, Fratta D, Tozzi A, Cardone A, Binkin N 1994. Prenatal toxoplasmosis screening in Campania region, Italy. It Gynaecol Obstet 6: 70-74.

EFSA Panel on Biological Hazards 2007. Surveillance and monitoring of Toxoplasma in humans, food and animals. EFSA J 583: 1-64.

EMSCOT - European Multicentre Study on Congenital Toxoplasmosis 2003. Effect of timing and type of treatment on the risk of mother to child transmission of Toxoplasma gondii. BJOG 110: 112-120.

Gilbert RE, Peckham CS 2002. Congenital toxoplasmosis in the United Kingdom: to screen or not to screen? J Med Screen 9: 135-141.

GURI 1995. Protocolli di accesso agli esami di laboratorio e di diagnostica strumentale per le donne in stato di gravidanza ed a tutela della maternità 1995. GURI 13.4, Serie generale n 87.

Lebech M, Joynson DHM, Seitz HM, Thulliez P, Gilbert RE, Dutton GN, Ovlisen B, Petersen E 1996. Classification system and case definitions of Toxoplasma gondii infection in immunocompetent pregnant women and their congenitally infected offspring. Eur $J$ Clin Microbiol Infect Dis 15: 799-805. 
Leroy V, Raeber PA, Petersen E, Rachid Salmi L, Kaminski M, Villena I, Buffolano W, Gilbert RE, EUROTOXO Group 2005. National public health policies and routine programs to prevent congenital toxoplasmosis, Europe. Available from: http://eurotoxo. isped.ubordeaux2.fr/WWW_PUBLIC/DOC/EUROTOXO_R1_ P3_European_national_policies_Dec2005.pdf.

SYROCOT - Systematic Review on Congenital Toxoplasmosis Study
Group 2007. Effectiveness of prenatal treatment for congenital toxoplasmosis: a meta-analysis of individual patient's data. Lancet 369: 115-122.

Thiebaut R, Leroy V, Alioum A, Binquet C, Poizat G, Salmi LR, Gras L, Salamon R, Gilbert R, Chene G 2006. Biases in observational studies of the effect of prenatal treatment for congenital toxoplasmosis. Eur J Obstet Gynecol Reprod Biol 124: 3-9. 